\title{
Primary ethmoid sinus squamous cell carcinoma in a young adult man
}

\author{
N Goncalves, MB BCh; L A Burnell, MB ChB, FCORL (SA); S Motakef, MB BCh, FCORL (SA); P C Modi, MB BCh, FCS (ORL) (SA) \\ Department of Otorhinolaryngology, Faculty of Health Sciences, University of the Witwatersrand, Johannesburg, South Africa
}

Corresponding author: $N$ Goncalves (drnicgoncalves@gmail.com)

In this case report we describe an aggressive primary ethmoid squamous cell carcinoma in an unusually young patient.

S Afr Med J 2016;106(9):893-894. DOI:10.7196/SAMJ.2016.v106i9.10575

Malignancies of the sinonasal cavity are uncommon, accounting for $3-5 \%$ of all malignancies of the head and neck. ${ }^{[1,2]}$ The predominant histological subtype of sinonasal tumours is sinonasal squamous cell carcinoma (SNSCC), accounting for up to $50-80 \%$ of all sinonasal malignancies. These most commonly occur in the maxillary sinus. ${ }^{[2]}$ The remaining $10-20 \%$ are intestinal-type adenocarcinoma, which mostly occur in the ethmoid sinus. ${ }^{[2]}$ There have been fewer than 500 reported cases of primary squamous cell carcinoma of the ethmoid sinus in the English literature. ${ }^{[3-15]}$ Sinonasal tumours typically present between the 5th and 6th decades of life..$^{[2]}$ Environmental exposures to wood and leather dust, as well as glues, formaldehyde, nickel and chrome, are strongly associated with the development of some sinonasal tumours. ${ }^{[2]}$

\section{Case report}

A 31-year-old black African man presented to our department with a 5-month history of swelling of the left side of the forehead, associated with a dull frontal headache and with progressive painless blindness of the left eye. The patient also reported a decrease in his sense of smell, along with loss of taste. There had also been an intermittent report of a bloodstained nasal discharge. The patient was an occasional smoker, with no occupational exposure to wood dust or industrial and textile compounds. $\mathrm{He}$ was HIV-negative. The physical examination revealed a $6 \times 5 \mathrm{~cm}$ diffuse, ill-defined, firm, mildly tender mass over the lower part of the left forehead. It appeared to be the head of a mushroom-type growth, with the stem arising through the nasion. The mass dominated over the left forehead with encroachment to the left medial epicanthal area, without causing epiphorea. There was intraorbital mass effect with proptosis and leftward and downward displacement of the globe, without ophthalmoplegia. His decreased visual acuity implied optic canal/ optic nerve involvement. There was no ptosis or chemosis. Anterior rhinoscopy revealed no intranasal sinus disease. Endoscopic middle meatus examination showed an illdefined ethmoid tumour mass not extending inferiorly. The drainage ostia of the maxillary sinus and anterior ethmoids were clear of disease. Endoscopic examination of the cribriform plate area was difficult owing to the distortion of the middle turbinate superiorly. Apart from cranial nerve I (loss of smell) and cranial nerve II (decreased visual acuity), all other cranial nerves were functionally intact.

A computed tomography (CT) scan showed a soft-tissue mass with contrast enhancement originating in the ethmoid sinus and extending superolaterally to the left side and infiltrating through the floor and posterior table of frontal sinus. There was also erosion through the cribriform plate. Destruction of the frontal sinus and frontal bone with intracranial extension into the frontal lobe was evident. Proptosis of the left orbit with destruction of the left bony orbital roof and lamina papyracea medially was noted. Magnetic resonance imaging (MRI) confirmed the mass as seen on the CT scan, with the tumour abutting and encasing the left extraconal optic nerve and with direct extension to the left frontal lobe (Fig. 1). A formal external open tissue biopsy via a Lynch incision was undertaken, under general anaesthesia.

The histopathological report was that of an invasive, moderately to poorly differentiated squamous cell carcinoma. Immunohistochemical staining, carried out to detect human papillomavirus and/or Epstein-Barr virus infection, was negative. Surgical resection was deemed contraindicated owing to the erosion of the tumour directly through

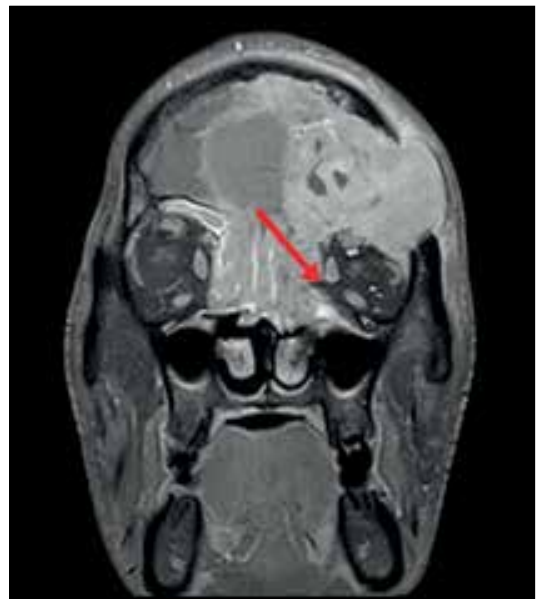

Fig. 1. T1 coronal MRI scan showing the epicentre of the tumour mass (arrow) in the ethmoid sinus with intracranial extension and erosion of frontal bone.

the cribriform plate to the frontal lobe, transgressing the cerebrospinal fluid space. Further treatment therefore involved radiation and cycles of chemotherapy.

\section{Discussion}

Primary ethmoid SNSCCs make up 5 - 25\% of all sinus malignancies. ${ }^{[10]}$ The literature is deficient on the precise incidence, as ethmoid tumours are often grouped with more common maxillary and nasal tumours. Squamous cell carcinomas are often grouped with other tumours, which may occur primarily within the ethmoid sinuses. ${ }^{[10]}$ While the average age of primary ethmoid SNSCC onset is known, only a few reports detailing a young age of onset are currently available in the published literature. The tumorigenesis of paranasal sinus tumours is unknown. Theories abound regarding certain chemical or viral agents that cause persistent or chronic inflammatory changes. ${ }^{[2]}$ Chronic inflammation in turn results in aberrant 
activation of tumour suppressor genes that support tumorigenesis. ${ }^{[2]}$ The above fails to explain the development of SNSCC in young patients or patients who have not been exposed to any known carcinogens. Early-onset tumorigenesis in young patients could be attributed to existing genetic precursor lesions and the accumulation of genetic alterations. ${ }^{[2]}$ The optimal care for patients with localised sinonasal carcinoma is complete surgical resection and postoperative radiotherapy. ${ }^{[2]}$ Ansa et al. ${ }^{[1]}$ showed the combination of surgery and radiation to be superior to radiation alone.

\section{Conclusion}

A complex interplay between genetic factors and yet-to-be-identified environmental triggers is likely to have contributed to the development of primary ethmoid SNSCC in an unusually young patient with a limited risk profile. Unveiling the molecular pathophysiology of SNSCC is likely to uncover potential molecular diagnostic markers and aid the identification of specific treatment modalities. 1. Ansa B, Goodman M, Ward K, et al. Paranasal sinus squamous cell carcinoma incidence and survival
based on surveillance, epidemiology, and end results data, 1973 to 2009. Cancer 2013;119(14):2602-
2610. DOI:10.1002/cncr.28108
2. Llorente JL, López F, Suárez C, Hermsen MA. Sinonasal carcinoma: Clinical, pathological, genetic
and therapeutic advances. Nat Rev Clin Oncol 2014;11(8):460-472. DOI:10.1038/nrclinonc.2014.97
3. Nicolai P, Castelnuovo P, Lombardi D, et al. Role of endoscopic surgery in the management of selected malignant epithelial neoplasms of the naso-ethmoidal complex. Head Neck 2007;29(12):1075-1082. DOI:10.1002/hed. 20636

4. Gras-Cabrerizo JR, Montserrat-Gili JR, León-Vintró X, et al. Treatment results for ethmoid sinus carcinoma. J Laryngol Otol 2009;123(10):1120-1124. DOI:10.1017/S0022215109990752

5. Islam S, Cole CV, Hoffman GR, Brennan PA. Bilateral axillary metastasis from a primary ethmoidal squamous cell carcinoma. J Laryngol Otol 2006;120(4):353-355. DOI:10.1017/S0022215106000375

6. Uchida D, Shirato H, Onimaru R, et al. Long-term results of ethmoid squamous cell or undifferentiated carcinoma treated with radiotherapy with or without surgery. Cancer J 2005;11(2):152-156.

7. Bhattacharyya N. Factors predicting survival for cancer of the ethmoid sinus. Am J Rhinol 2002;16(5):281-286.

8. Tiwari R, Hardillo JA, Tobi H, Mehta D, Karim AB, Snow G. Carcinoma of the ethmoid: Results of treatment with conventional surgery and post-operative radiotherapy. Eur J Surg Oncol 1999;25(4):401-405. DOI:10.1053/ejso.1999.0665

9. Waldron JN, O'Sullivan B, Warde P, et al. Ethmoid sinus cancer: Twenty-nine cases managed with primary radiation therapy. Int J Radiat Oncol Biol Phys 1998;41(2):361-369. DOI:10.1016/S03603016(98)00018-2

10. Kraus DH, Sterman BM, Levine HL, et al. Factors influencing survival in ethmoid sinus cancer. Arch Otolaryngol Head Neck Surg 1992;118(4):367-372. DOI:10.1001/archotol.1992.01880040025005

11. Morokhoev VI. [Errors in the early diagnosis of malignant tumors of the ethmoid sinus]. Vestn Otorinolaringol 1990;(5):60-64

12. Lavertu P, Roberts JK, Kraus DH, et al. Squamous cell carcinoma of the paranasal sinuses: The Cleveland Clinic experience 1977 - 1986. Laryngoscope 1989;99(11):1130-1136. DOI:10.1288/00005537198911000-00005

13. Spiro JD, Soo KC, Spiro RH. Squamous carcinoma of the nasal cavity and paranasal sinuses. Am J Surg 1989;158(4):328-332.

14. Lampe HB, St Pierre S, Baker SR. Carcinoma of the ethmoid sinus. Am J Otolaryngol 1986;7(3):209212.

15. Badib AO, Kurohara SS, Webster JH, Shedd DP. Treatment of cancer of the paranasal sinuses. Cancer 1969;23(3):533-537.

Accepted 24 January 2016. 